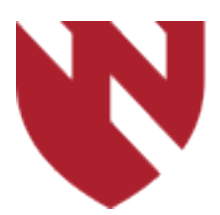

\title{
Expanded Mutation Profiling in Appendix Peritoneal Metastasis Has Prognostic and Therapeutic Utility When Managed with Cytoreductive Surgery/ Hyperthermic Intraperitoneal Chemotherapy (CRS/HIPEC)
}

Chunmeng Zhang

University of Nebraska Medical Center

Ben Plambeck

University of Nebraska Medical Center

Maggie Moore

University of Nebraska Medical Center

Alexander Tu

University of Nebraska Medical Center

Ryan Miku

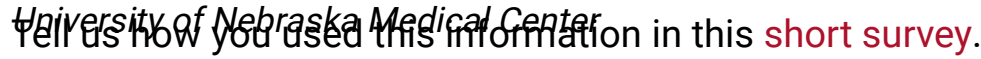

Follow this and additional works at: https://digitalcommons.unmc.edu/gmerj

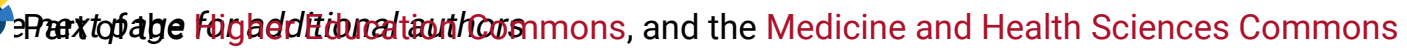

\section{Recommended Citation}

Zhang, C., Plambeck, B., Moore, M., Tu, A., Miku, R., Shostrom, V., Brown, K., Cushman-Volkoun, A., Swanson, B., , Foster, J. Expanded Mutation Profiling in Appendix Peritoneal Metastasis Has Prognostic and Therapeutic Utility When Managed with Cytoreductive Surgery/ Hyperthermic Intraperitoneal Chemotherapy (CRS/HIPEC). Graduate Medical Education Research Journal. 2020 Sep 29; 2(1). https://digitalcommons.unmc.edu/gmerj/vol2/iss1/31 


\section{Expanded Mutation Profiling in Appendix Peritoneal Metastasis Has Prognostic and Therapeutic Utility When Managed with Cytoreductive Surgery/ Hyperthermic Intraperitoneal Chemotherapy (CRS/HIPEC)}

\section{Creative Commons License}

\section{(c) (†) $\Theta$}

This work is licensed under a Creative Commons Attribution-Noncommercial-No Derivative Works 4.0 License.

\section{Authors}

Chunmeng Zhang, Ben Plambeck, Maggie Moore, Alexander Tu, Ryan Miku, Valerie Shostrom, Krista Brown, Allison Cushman-Volkoun, Benjamin Swanson, and Jason Foster 
caveat phrases in the original radiology interpretation. The studies were categorized into diagnostic, technically limited, or nondiagnostic studies.

Results: Studies performed using ASIR-V (375.6 $\left.\mathrm{mGy}^{*} \mathrm{~cm}[255.9,501.4]\right)$ had a significantly lower DLP compared to those using FBP (695.3 mGy*cm [410.6, 1110.0]). The diagnostic quality was superior when performed using ASIR-V, with 90\% (481/533) of studies being diagnostic, compared to $80 \%$ $(452 / 562 ; \mathrm{P}<.0001)$ in the FBP group.

Conclusions: CTPA performed using ASIR-V both reduced radiation dose as well as improved diagnostic confidence compared to CTPA performed using FBP.

https://doi.org/10.32873/unmc.dc.gmerj.2.1.029

\section{References}

1 Moore, Alastair JE, et al. "Imaging of acute pulmonary embolism: an update." Cardiovascular Diagnosis and Therapy 8.3 (2018): 225.

2 Roshkovan, Leonid, and Harold Litt. "State-of-the-art imaging for the evaluation of pulmonary embolism." Current Treatment Options in Cardiovascular Medicine. 20.9 (2018): 71

3 Lenga, Lukas, et al. "Single-and dual-energy CT pulmonary angiography using second-and thirdgeneration dual-source CT systems: comparison of radiation dose and image quality." European Radiology 29.9 (2019): 4603-4612.

4 Singh, Sarabjeet, et al. "Adaptive statistical iterative reconstruction technique for radiation dose reduction in chest CT: a pilot study." Radiology. 259.2 (2011): 565-573.

5 Kwon, Heejin, et al. "The adaptive statistical iterative reconstruction-V technique for radiation dose reduction in abdominal CT: comparison with the adaptive statistical iterative reconstruction technique." The British Journal of Radiology. 88.1054 (2015): 20150463.
6 Qiu, D., and E. Seeram. "Does iterative reconstruction improve image qual-ity and reduce dose in computed tomography." Radiol Open J. 1.2 (2016): 42-54.Desai

7 Gaurav S., et al. "Impact of iterative reconstruction on image quality and radiation dose in multidetector CT of large body size adults." European Radiology. 22.8 (2012): 1631-1640.

\section{Expanded Mutation Profiling in Appendix Peritoneal Metastasis Has Prognostic and Therapeutic Utility When Managed with Cytoreductive Surgery/Hyperthermic Intraperitoneal Chemotherapy (CRS/HIPEC)} Chunmeng Zhang ${ }^{1}$, Ben Plambeck², Maggie Moore ${ }^{2}$, Alexander Tu², Ryan Miku², Valerie Shostrom ${ }^{3}$, Krista Brown ${ }^{4}$, Allison Cushman-Volkoun ${ }^{5}$, Benjamin Swanson ${ }^{5}$, Jason Foster ${ }^{1}$

${ }^{1}$ University of Nebraska Medical Center, Department of Surgery

2University of Nebraska Medical Center, College of Medicine

${ }^{3}$ University of Nebraska Medical Center, College of Public Health, Department of Biostatistics

${ }^{4}$ University of Nebraska Medical Center, College of Public Health, Department of Epidemiology

${ }^{5}$ University of Nebraska Medical Center, Department of Pathology

Mentor: Jason Foster

Program: General Surgery

Type: Original Research

Background: The identification of relevant biological pathways and mutations is integral to improving outcomes in appendix peritoneal metastases (APM). Interrogation of cancers with Next Generation Sequencing (NGS) 50-gene mutation panels has become more widely utilized identifying prognostic and actionable mutations. This study is a dedicated analysis of the value of expanded mutation analysis in APM.

Methods: The IRB approved study included 51 APM patients where data was retrospectively collected from a CRS/HIPEC registry treated 2012-2018. Standard clinical 50 -gene NGS analysis was performed in CLIA approved lab. All patients underwent CRS/ HIPEC with mitomycin $\mathrm{C}$ delivered for 90 minutes at 41-42 C. Peritoneal Cancer Index (PCI), Completeness of Cytoreduction (CC) score, length of stay, progression free survival (PFS), overall survival (OS) were collected along with the rates and types of mutation in APM. OS and PFS analyses were performed on all, high grade (HG), and low grade (LG) APM, specifically evaluating the impact of smad4 and p53 mutations on survival.
Results: Eighty-four percent of APM had a mutation identified with $58 \%$ of cases harboring $\geq 2$ mutations. Kras was most frequent, $66 \%$ of APM (88\% LG 44\% HG) and GNAS identified in $88 \%$ of LG APM. Smad 4 or p53 mutation occurred in $25 \%$ of APM and a significant reduction in OS in all APM ( 22 vs 88 months $\mathrm{p}=0.0026)$ and HG APM (20 vs 47 months $\mathrm{p}=0.0502$ ) was observed. Smad4 mutation was also associated with a significant reduction in PFS APM ( $p=0.0192)$. Actionable mutations were identified in $70 \%$ of APM.
Conclusion: Smad4 and p53 mutations were associated with more aggressive APM and maybe a useful tool in patient selection and outcome. Expanded mutation profiles is valuable in APM and further application is warranted. Research in Kras, p53 and smad4 pathways and drug development will benefit APM.

https://doi.org/10.32873/unmc.dc.gmerj.2.1.030

\section{Table 1.}

p53 and/or SMAD4 mutations are associated with significantly reduced overall survival (OS) in all patients and high grade (HG) subgroup; SMAD4 mutations alone was associated with significantly shortened progression free survival (PFS) in all patients and high-grade subgroup. LG, low grade.

\begin{tabular}{lccc} 
& $\begin{array}{c}\text { WT P53 \& SMAD4 } \\
\text { (months) }\end{array}$ & $\begin{array}{c}\text { +P53 OR +SMAD4 } \\
\text { (months) }\end{array}$ & P VALUE \\
\hline OS $_{\mathrm{ALL}}$ & 88 & 22 & $<0.01$ \\
\hline OS $_{\mathrm{HG}}$ & 47 & 20 & 0.05 \\
\hline & $\begin{array}{c}\text { WT SMAD4 } \\
\text { (months) }\end{array}$ & 10 & $\begin{array}{c}\text { +SMAD4 } \\
\text { (months) }\end{array}$ \\
\hline PFS $_{\mathrm{ALL}}$ & 6.5 & 3.5 & 0.02 \\
\hline PFS $_{\mathrm{HG}}$ & 20 & 1.5 & 0.01 \\
\hline $\mathrm{PFS}_{\mathrm{LG}}$ & & 7.5 & 0.09
\end{tabular}

